\title{
Preferencias y significado de valores en estudiantes del bachillerato
}

Osorto, Santos Federico; Soriano, Oscar Alberto

Preferencias y significado de valores en estudiantes del bachillerato

Revista Educación, vol. 46, núm. 1, 2022

Universidad de Costa Rica, Costa Rica

Disponible en: https://www.redalyc.org/articulo.oa?id=44068165032

DOI: https://doi.org/10.15517/revedu.v46i1.45402

\section{(c) $(1) \Theta(9)$}

Esta obra está bajo una Licencia Creative Commons Atribución-NoComercial-SinDerivar 3.0 Internacional. 
Artículos Científicos

\title{
Preferencias y significado de valores en estudiantes del bachillerato
}

\author{
Preferences and Meaning of Values among High School Students \\ Santos Federico Osorto \\ Universidad Politécnica de Honduras, Honduras \\ santos.rodriguez@uph.edu.hn \\ (iD) https://orcid.org/000-0002-3288-1557 \\ Oscar Alberto Soriano \\ Universidad Pedagógica Nacional Francisco Morazán, \\ Honduras \\ soriano_hn@yahoo.com \\ iD https://orcid.org/0000-0002-2576-0941
}

DOI: https://doi.org/10.15517/revedu.v46i1.45402 Redalyc: https://www.redalyc.org/articulo.oa? $\mathrm{id}=44068165032$

Recepción: 09 Febrero 2021

Aprobación: 08 Abril 2021

\section{Resumen:}

Este artículo resume la investigación realizada para conocer las preferencias de valor y el significado que el estudiantado otorga a los valores. El estudio se desarrolló en los centros educativos del área urbana de Choluteca, Honduras. La metodología aplicada fue de diseño mixto. El acercamiento cuantitativo se desarrolló con el Portrait Values Questionnaire (PVQ) de la Teoría Refinada de Valores Humanos de Shalom Schwartz y el acercamiento cualitativo con un diseño fenomenológico-interpretativo de los valores; se utilizó la entrevista semiestructurada como técnica de recolección de información. Los resultados muestran que el universalismopreocupación encabeza las preferencias del estudiantado, es decir, la preocupación por la búsqueda de igualdad, justicia y protección de las demás personas. El hedonismo presenta diferencias en la variable edad. La búsqueda de poder es lo menos preferido. La humildad presenta una ambivalencia demostrada con los hallazgos de los dos acercamientos, su significado radica en ser constructor de modelos de vida, sinónimo de bondad y necesario para la vida profesional. El anhelo por ser profesionales al servicio de las demás personas caracteriza el interés por los valores del estudiantado participante. Como conclusión se resalta el interés por las demás personas que tiene el estudiantado, la réplica de la correlación del hedonismo con la edad, la humildad como valor anhelado en la vida profesional y la religiosidad no practicante como forma de vida y fuente de valores. Se recomienda buscar correlaciones entre la propuesta de Schwartz y comportamientos específicos en el contexto hondureño, además de ampliar los estudios cualitativos sobre valores con poblaciones más extensas.

Palabras clave: Valores, Preferencias, Humildad, Ambivalencia, Estudiantes.

\section{Abstract:}

This article summarizes the findings of a study on value preferences among students and the meaning they attribute to these values. The study was conducted at various schools located in the urban regions of Choluteca in southern Honduras. The study methodology consisted of a mixed design using the Portrait Value Questionnaire (PVQ) of the Refined Theory of Human Values by Shalom Schwartz to provide a quantitative approach. A phenomenological-interpretive design based on semi-structured interviews contributed data for a more qualitative approach. Based on the results, student preferences leaned towards a concern for universalism, which entails a search for equality, justice and protection of others, whereas hedonism varied according to age. There was a lower preference with regards to ambition and power. Humility reflected student ambivalence, as demonstrated with findings from both approaches. Humility is synonymous with goodness and an important value for one's work life. Education and achieving a professional career path to serve others was a characteristic value among students. According to the conclusion, students possess an overall interest in the other which emphasizes the correlation between hedonism and age. Humility was viewed as a desired value, synonymous of goodness and particularly helpful and desired for one's professional life. Non-practicing religious beliefs also served as an indicator of moral values. We recommend finding correlations between Schwartz's proposal and specific behaviors within the context of Honduras, as well as expand these studies to areas with higher population densities.

KEYwORDs: Values, Preferences, Humility, Ambivalence, Students. 


\section{INTRODUCCIÓN}

Actualmente la referencia a los valores es abundante. Existen instituciones que se lanzan a proponer decálogos, con los cuales manifiestan identificarse. Desde la educación se propone una formación integral inspirada en valores. La sociedad, en general, parece tener interés en los valores y se habla de ellos de modo persistente en los momentos de crisis social. Ya desde el siglo pasado, un escrito sobre Ortega y Gasset (1961) recuerda "que la preocupación teórica y práctica en torno a los valores es uno de los hechos más hondamente reales del tiempo nuevo" (p. 315). Las continuas referencias a los valores es mencionado por Adela Cortina, cuando afirma que los valores están de actualidad, pues "alguna especial circunstancia invita a sacar a la palestra lo que es un elemento constitutivo de nuestra realidad personal” (Cortina, 2009, p. 18).

Pero en el contexto hondureño son pocas las investigaciones en axiología, esto se convierte en un vacío al momento de buscar referentes empíricos, en consideración con la preocupante situación que padece la población joven. Por ejemplo, el ambiente de violencia es uno de los temas que ocupa la atención de organismos internacionales como el Alto Comisionado de las Naciones Unidas para los Refugiados [ACNUR] (2018), para quien "entre el 2010 y el 2018 se registraron 47,366 homicidios y los más afectados son los hombres en el rango de 15 a 30 años de edad” (p. 9). El nivel de violencia ha crecido tanto que ya existe un desplazamiento interno forzado aparte de la migración irregular que tiene su origen en situaciones de violencia y de pobreza, que es otra situación crítica. Según la Comisión Económica para América Latina [CEPAL] (2019), en cifras de 2015 a 2018 "el $61.9 \%$ de la población vive en pobreza y el $38.7 \%$ se encuentra en pobreza extrema" (p. 100).

En este contexto de problemas sociales complejos, es pertinente indagar cuáles son las preferencias de valor de las personas adolescentes y si los valores tienen algún significado para ellas, en consideración de que se encuentran en una etapa formativa de transición entre la educación media y la universidad. Al graduarse en la secundaria, ¿cuáles son las preferencias de valor y qué significado otorgan a los valores el estudiantado adolescente cuando egresa de la educación media y llega a las aulas universitarias?

Después de hacer referencia al contexto de la población estudiantil con la que se trabajó, se presenta el marco teórico que aborda el tema de los valores desde la reflexión filosófica, reflexiones sobre la moral, los valores y la reflexión ética. Se mencionan propuestas de búsqueda de valores mediados por disciplinas como la neurociencia, la etología y los estudios gestados desde la psicología, de los cuales sobresale la propuesta de Schwartz en los tres momentos, según se indica. Continúa la descripción metodológica, el instrumento utilizado, la descripción de la población y las técnicas utilizadas. Después se exponen los resultados relevantes encontrados, se agrega una discusión sobre estos, para llegar a las conclusiones y una mención general de las recomendaciones.

\section{MARco TEÓRICo}

La reflexión sobre los valores nació con la filosofía. Autores como Sandoval (2007) y Benítez (2015) han visto los primeros intentos en los diálogos de Platón: Hipias mayor, Laques, Banquete, Fedro, y en la obra de Aristóteles (Ética a Nicómaco), donde se encuentran las primeras reflexiones axiológicas o una prototeoría sobre los valores.

Pero es Frondizi (1972) el heredero de toda una tradición que vio el amanecer en autores como Ortega y Gasset, de quien retoma ideas y contesta a la pregunta: ¿qué son los valores? Dice de ellos que "no son cosas, ni elementos de cosas, sino propiedades, cualidades sui generis que poseen ciertos objetos llamados bienes" (Frondizi, 1972, p. 17). También señala que "los bienes equivalen a las cosas valiosas, esto es, a las cosas más el valor que se les ha incorporado" (Frondizi, 1972 p. 15). Los valores dependen de los bienes para existir, tienen una vida parasitaria. Pero, ¿las cosas tienen valor porque se prefieren o se prefieren porque tienen valor? 
Las respuestas a esta pregunta iniciaron y fomentaron extensas discusiones axiológicas por buen tiempo, con ello se dio inicio al antagonismo filosófico entre objetivismo y subjetivismo.

Por otro lado, es de resaltar dos características de los valores, mencionados por Cortina (2009). La primera hace referencia a la fase atrayente del valor y lo repulsivo del antivalor. La segunda se refiere al hecho de la superioridad en cómo se presentan algunos valores sobre la inferioridad de otras personas. Esto tiene que ver con las preferencias individuales. Tampoco se deben olvidar los aportes de la obra de Fullat (2005), quien plantea que el homo occidentalis ha sido construido por el homo cristianis. El proceso dialéctico emanado de tres mundos, el griego, el romano y el de Jerusalén, ha sido el hontanar de donde brota toda la cosmovisión axiológica de Occidente. Valores como la humildad habrían visto su amanecer en el ambiente cristiano como producto de este proceso. Desde lo mencionado, se puede afirmar que la reflexión filosófica sobre los valores cuenta ya con una buena trayectoria abundante en novedad y diversidad de propuestas.

\section{Las reflexiones sobre la moral}

El interés por la reflexión sobre los valores ha estado acompañado por la enseñanza de la moral y la reflexión ética, especialmente con las nuevas generaciones. Las propuestas de educación moral se han realizado desde la ética aplicada, la cual "se ocupa de reflexionar sobre la presencia de principios y orientaciones morales en los distintos ámbitos de la vida social y constituye de algún modo la aplicación de una moral cívica a cada uno de esos ámbitos" (Cortina, 2013, p. 1). Algunas de las cuestiones que se suscitan giran en torno a si la moral puede enseñarse, si es así, ¿̇cuáles serían las metodologías adecuadas para la enseñanza de la moral? De igual modo, en la reflexión aparece el debate entre los postulados de orientación normativa contra la orientación basada en "los análisis de la acción humana, del sujeto moral y de la racionalidad práctica" (Thiebaut, 2009 p. 606), lo cual amplía el análisis de los comportamientos morales al integrar las emociones y los sentimientos. En el espacio educativo, la pregunta que recoge esa disyuntiva puede plantearse así: ¿es una clase la mejor forma de aportar reflexiones sobre la moral o más bien debería estudiarse lo que se considera buenos ejemplos que aportan modelos de vida?

En la perspectiva normativa se pueden entender a los valores éticos como ideales de conducta para la vida en sociedad. Entre esos valores éticos se contaría con algunos considerados muy generales y fundantes de las posibilidades de la convivencia humana y en colectivos específicos. Entre ellos están la justicia, honestidad, tolerancia, responsabilidad, imparcialidad, empatía, respeto, compromiso, solidaridad, beneficencia, altruismo, etc. En cambio, en el campo de la moral, en tanto que normas o guías de conducta para las personas, desde su individualidad y con el supuesto de una autonomía relativa, pues solamente en sociedad y en relación con sus semejantes cada ser perfila su particularidad individual, se encuentran los preceptos morales, como reglas asumidas e interiorizadas. Por ejemplo, tratar a las demás personas con el mismo trato que se quiere recibir; respetar las instituciones; aplicar los códigos de comportamiento en el ámbito donde cada quien se encuentre; proteger a la población más vulnerable; cuidar los espacios y bienes públicos; no robar; no mentir, etc.

La mención de valores éticos y la referencia a los preceptos morales simplemente indica una problemática más compleja surgida de la tensión entre la perspectiva comunitaria frente a la perspectiva liberal en torno a la educación en valores y la formación de la conciencia moral de la ciudadanía (Naval, 2001; Rorty, 2004; Cortina, 2009; Camps, 2010).

La ética de la responsabilidad (Jonas, 1995) es un enfoque que debe registrarse, como antecedente a tener en cuenta para hilvanar propuestas éticas en la formación de la nueva ciudadanía. Sus planteamientos surgen de la constatación de los impactos del desarrollo científico-tecnológico en la vida humana durante el siglo $\mathrm{XX}$, con la exigencia de cambios en los presupuestos fundamentales de la ética, a fin de establecer "una ética de nueva planta basada en otros valores (temor, austeridad, respeto o contención), todos ellos entrelazados a fin de preservar la integridad del mundo frente a los posibles e innumerables abusos de poder" (Turró 2001, 
p. 241). A partir de esa premisa desde el enfoque de la ética de la responsabilidad se indica que "la humildad, el respeto y la moderación van a ser virtudes con qué contar en nuestra marcha” (Turró 2001, p. 242).

En vistas a la formación de la personalidad moral, el proceso de la educación moral, concerniente a cada ser humano, consciente, libre y responsable, implica, en primer lugar, alejarse de toda coacción externa. De lo contrario no habría libertad. Por lo tanto, una moral que esté lejos de cualquier imposición religiosa, o de cualquier otra índole, calificaría para los "elementos esenciales de la moralidad que son: el espíritu de disciplina, la adhesión a los grupos sociales y la autonomía de la voluntad” (Puig, 2003, p. 23). Las propuestas de Piaget (1984) y Kohlberg (1992) testifican la reflexión que tiene la búsqueda de la libertad como meta.

En la actualidad, se desarrollan investigaciones en disciplinas que, como las neurociencias y la antropología, aportan datos y enriquecen la reflexión. Un ejemplo son los paralelismos encontrados en otras especies de animales no humanos, a partir de observaciones y experimentos desarrollados (De Waal, 2014). Uno de ellos es la empatía, el hecho de sentir el dolor ajeno, de solidarizarse compasivamente. El altruismo parece iniciar con el cuidado maternal, desde allí penden todos los demás elementos que podrían considerarse morales en otras especies. Las neuronas espejo se descubrieron en investigaciones con primates, tal como lo plantea Iocoboni (2009); esto abrió las posibilidades de estudios empíricos, que incluso estimularon investigaciones empíricas con humanos y humanas.

De tal manera, la reflexión axiológica dejó de ser algo exclusivo de la filosofía, pues se amplió y enriqueció con aportes de la antropología, la sociología y la psicología. Con la Figura 1 se pretende representar el puente que va desde la reflexión filosófica hasta los estudios de campo en la psicología a partir de cinco personas autoras, cuyo elemento en común ha sido el estudio de los valores. La motivación por representarlo de esta manera es meramente expositiva.

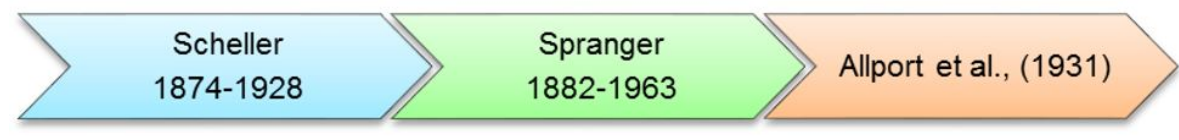

FIGURA 1.

De la reflexión filosófica a los estudios de campo.

Fuente: elaboración propia.

Fue Scheller (2001) el primero en hacer una propuesta de valores desde la filosofía. Representada en una pirámide, se constituía como un imperativo, es decir, esos eran los valores que deberían ordenar la conducta de todas las sociedades. Esta posición débil como propuesta, contrasta con los cambios producidos en las sociedades y la misma dinámica de la historia. Por tanto, las nuevas propuestas se enfocan en tipos de hombre definidos a partir de sus preferencias. Para el caso, Spranger (1928) propuso seis tipos de personas y esta propuesta fue llevada al terreno empírico por Allport et al. (1931). Este trabajo se convirtió en uno de los primeros test desarrollado para investigaciones sobre valores.

En las últimas tres décadas del siglo XX, aparecieron nuevos aportes también desde la psicología. De ellos se pueden mencionar a Rokeach (1973), quien propuso la teoría de valores instrumentales y finales; Inglehart (1994), impulsor de la Encuesta Mundial de Valores; Triandis (2001), y Hofstede (2011). También el psicólogo social de la Universidad Hebrea de Jerusalén, Shalom Schwartz, propone una teoría transcultural sobre los valores, esta fue expuesta en tres momentos (1987, 1992 y 2012).

\section{¿Qué son los valores para Schwartz?}

Para Schwartz, los valores son conceptos o creencias: una persona, sin importar de qué se trate, lo que cree estará por encima de lo que se le pide y si siente sus valores amenazados, debatirá y sabrá que su dignidad está en juego. Sobre un fin deseado: los valores aspiran a todas las metas trascendentales para la comunidad (la justicia, la solidaridad, la fortaleza, la templanza, etc.). Trascienden las situaciones específicas: la práctica de los valores 
va más allá de una simple actitud hacia algo específico. Si alguien actúa con justicia, sus acciones hablarán de esta persona en cualquier ambiente. Guían la selección o evaluación de comportamientos: "Evaluamos personas, comportamientos, eventos, positivamente, si promueven o protegen el logro de los objetivos que valoramos" (Schwartz 2012, p. 16). Están ordenados según la importancia relativa: "Cuando pensamos en nuestros valores, pensamos en lo que es importante para nosotros en la vida" (Schwartz 2012, p. 3) Son estándares o criterios de vida: al final, el conjunto de valores en la persona guía sus acciones y le da horizonte a su vida.

La teoría de Schwartz está construida sobre tres requerimientos universales: a) necesidades biológicas del organismo, b) requisitos interactivos para la coordinación interpersonal, c) demandas sociales para el bienestar y la supervivencia del grupo (Schwartz y Bilsky, 1987, p. 550).

\section{Sobre la teoría de Schwartz}

En esta teoría, se concibe los valores como bases motivacionales para el comportamiento. El autor descubrió que existen tipos motivacionales de valor, esto es, motivaciones intrínsecas a las personas que contienen, a su vez, en sus rasgos, preferencias de valores que se replican en muchas culturas. El planteamiento original lo basó en estudios realizados por Rokeach (1973), de él retomó los siete tipos motivacionales de valor de su primera propuesta: el prosocial, la conformidad restrictiva, el placer, el logro, la madurez, la autodeterminación y la seguridad.

Después de estudios en diferentes países, el autor revisó su teoría y el número de tipos motivacionales se elevó a diez. Lo prosocial pasó a ser benevolencia, la conformidad restrictiva pasó a conformidad, el placer a hedonismo y la madurez a universalismo. Además, se sumaron tradición, estimulación y poder, este último muy fuerte en culturas como las orientales. Por otro lado, en la teoría de Schwartz, hay dos aspectos que resultan clave: la adyacencia y la polaridad de los tipos motivacionales de valor. La adyacencia es la cercanía de unos valores con otros en sus preferencias y la polaridad es el antagonismo de unos valores con otros. Todo se organiza en un círculo motivacional, un continuum dinámico. Las culturas se diferencian por preferir unos valores; esta preferencia también supone oposición o negación con los valores opuestos.

Finalmente, después de críticas que recibió el instrumento original y los tipos motivacionales que medía, en el año 2012 surge la Teoría Refinada con 19 tipos motivacionales de valor. La seguridad se dividió en dos: personal y social; la benevolencia en cuidado y fiabilidad; la conformidad con las reglas y entre personas; la autodirección en pensamiento y acción; el universalismo en preocupación, tolerancia y naturaleza; el poder en dominación y recursos de poder. También se agregó la humildad y la imagen. En general, la teoría, además de replicarla en diversas sociedades, varias personas autoras también han encontrado correlaciones con algunos comportamientos específicos, según se muestra en Moreno (2006); Kasof et al. (2007); Goodwin et al. (2002), por mencionar algunas.

En la Tabla 1 se expone el proceso evolutivo de la teoría. Se enfatiza cómo, a partir del primer momento, algunos dominios reciben diferentes nombres, otros no cambian y en cada momento van apareciendo otros que no estaban contenidos en el momento de la teoría que antecede. En el tercer momento, la mayoría de estos se subdividen en dos y hasta tres en el caso del universalismo, tal como se ha descrito. 
TABLA 1

Evolución de la teoría de Schwartz

\begin{tabular}{lll} 
Primer momento & Segundo momento (Teoría revisada) & Tercer momento (Teoría refinada) \\
\hline Seguridad & Seguridad & Seguridad personal \\
& & Seguridad social \\
Prosocial & Benevolencia & Benevolencia-cuidado \\
& & Benevolencia-fiabilidad \\
Conformidad restrictiva & Conformidad & Conformidad con las reglas \\
& & Conformidad entre personas \\
Placer & Hedonismo & Hedonismo \\
Logro & Logro & Logro \\
Autodeterminación & Autodirección & Autodirección-pensamiento \\
Madurez & Aniversalismo & Universalismo-preocupación \\
& & Universalismo-tolerancia \\
& & Universalismo-naturaleza \\
& Poder & Poder-dominación \\
& & Recursos de poder \\
& Tradición & Tradición \\
& Estimulación & Estimulación \\
\hline & & Humildad \\
& & Imagen \\
\hline
\end{tabular}

Fuente: elaboración propia a partir de Schwartz y Bilsky (1987); Schwartz (1992); Schwartz (2012).

\section{Metodología}

Se optó por un estudio mixto sobre los valores. Se asume que el "método mixto es la integración sistemática de los métodos cuantitativo y cualitativo en un solo estudio con el fin de obtener una fotografía más completa del fenómeno" (Cedeño, 2012, p. 19). La adopción de este diseño surge a partir de los objetivos específicos planteados, con los cuales se buscaba, en primer lugar, identificar las preferencias de valor en función de la importancia otorgada por la población estudiantil, que, a su vez, llevó al análisis de las diferencias presentadas según algunas variables como sexo, edad, naturaleza (confesionales-aconfesionales) y tipo de centro (públicoprivado). La dimensión cualitativa surge del planteamiento: describir el significado que el estudiantado otorga a los valores a partir de su formación humana e interpretar el interés del estudiantado por los valores para su vida profesional.

Para el acercamiento cuantitativo se aplicó un diseño no experimental, transversal, con un alcance descriptivo para entender el fenómeno y hay una sola variable: tipos motivacionales de valor. La población estudiantil con la cual se trabajó cursaba el último año en Ciencias y Humanidades de los centros educativos que se ubican en el área urbana de Choluteca, Honduras. La investigación se desarrolló con 457 participantes distribuidos en los 16 centros educativos, entre ellos: públicos y privados, confesionales y aconfesionales, del sistema presencial y a distancia. Es decir, se encuestó a toda la población. Se concluyó que era viable hacerlo de esa manera. En primer lugar, se confrontó el registro de la Dirección Municipal, la matrícula de cada centro educativo y la población real en las aulas que difería sobremanera con la primera, en consideración con el impacto del fenómeno de la migración en el sistema educativo hondureño. Se aplicó el Cuestionario de Valores Retratados (PVQ), el cual fue adaptado al español por Sánchez (2016); tropicalizado para el contexto hondureño y recomendado por el Dr. Schwartz. Este instrumento mide 19 tipos motivacionales de valor contenidos en cuatro dimensiones que son: autotrascendencia, que contiene la benevolencia (fiabilidad y cuido) y el universalismo (preocupación, tolerancia y naturaleza); el auto crecimiento, que contiene el poder (dominación y recursos de poder), la imagen y logro. La apertura al cambio, que contiene libertad (acción y pensamiento) estimulación y hedonismo. Finalmente la conservación, que contiene la seguridad social y la 
seguridad personal, la tradición y la conformidad (con las reglas y con las demás personas). Se buscaba que, a partir de la descripción de una persona, la población joven encuestada manifestara qué tan parecidos son con esos rasgos que se les presentaban en una escala, en la cual el número 1 significa no separece absolutamente nada a mi y el número 6 indica muy parecido a mí. Es decir, los ítems remitían al estudiantado a ciertas conductas que, para la persona a la que se le presenta, son preferibles o deseables. Al final, se obtiene un "autorreporte de similitud y de allí se infieren los valores personales del encuestado” (Imhoff y Brussino, 2013, p. 138).

El análisis de la información en el acercamiento cuantitativo se hizo utilizando estadística descriptiva, específicamente .como referente. También se hizo uso de la $t$ de student para comparar medias de dos grupos y ANOVA de un factor para más de dos grupos. Pero los valores

son cualidades no reales, es decir, que no se pueden cuantificar ni medir, se estiman, e involucran la subjetividad de la persona en lo que se refiere a la apreciación establecida con relación al grado de valor que presenta una realidad específica (Medina, 2007, p. 381).

Por ello, la parte cualitativa es un imperativo. Se desarrolló esta parte con un diseño fenomenológico. Se considera a Edmund Husserl (1859-1938) el gran exponente de la fenomenología como corriente filosófica. Como método, la descripción fenomenológica se apoya en lo experimentado, lo vivido, lo interno y la percepción que sobre su manifestación hace la persona cuando interactúa con sus semejantes. Por lo tanto, una manera de acceder a la fenomenología de las partes participantes es mediante el estudio del discurso explicitado por ellas con respecto al fenómeno bajo estudio (Gutierrez, 2016). La fenomenología explora esas realidades que no son muy comunicables, pero esenciales para entender la vida de una persona. Es a partir de aquí que la descripción es sumamente importante. Una descripción que surge de la comprensión e interpretación de las vivencias individuales o colectivas. Por medio de este proceso se revela lo que subyace y da sentido a los actos externos de cada persona.

Se trata de ver el fenómeno de los valores a partir del "punto de vista de otras personas, describiendo, comprendiendo e interpretando" (Rodríguez et al., 1999, p. 42), quien investiga intenta ver las cosas desde otros puntos de vista. El resultado final "será una descripción en una narración que dibujará un modelo de las invariantes estructurales de un determinado tipo de experiencias” (p. 42). Experiencias vividas prereflexivamente por la población estudiantil del último año del bachillerato en Ciencias y Humanidades, en la cotidianidad e inmediatez propias de su mundo y vida colegial. Para el proceso del diseño fenomenológico se tomó como modelo el trabajo de Escalante (2010) y se desarrolló en cuatro etapas:

1-Etapa previa o clarificación de presupuestos: se partió de los siguientes presupuestos: existe una vivencia de valores, experiencias mostradas a partir de la práctica de estos por parte de la población estudiantil, modelos de personas que practican valores y, por tanto, se convierten en ejemplos a seguir.

2- Recoger la experiencia vivida: se eligió la técnica de recolección de datos: la entrevista semiestructurada. Se desarrollaron cinco entrevistas individuales y tres grupos focales con los cuales se llegó a la saturación en las respuestas. El muestreo fue intencionado. Se utilizó como criterio de selección el impacto ocasionado por el cuestionario cuantitativo aplicado. Esto es, después de contestar el cuestionario, algunas y algunos estudiantes compartían comentarios sobre este. En esos casos, se le invitaba al grupo focal cuando eran varias personas o a la entrevista individual cuando eran pocas. Las bitácoras de campo ayudaron a profundizar más sobre el tema. Según F. Robles (comunicación personal, 20 de febrero de 2018), la entrevista cualitativa "se define como una reunión para conversar e intercambiar información entre una persona y otra u otras". En consideración de que el problema de estudio es muy difícil de observar, se acudió a esta técnica en las modalidades mencionadas.

3- Reflexionar acerca de la experiencia vivida-etapa estructural: al tomar como base esa información, se interpretó el significado de los valores a partir de la formación humana y el interés por estos para la vida profesional.

4- Escribir y reflexionar acerca de la experiencia vivida: es la búsqueda de sentido y de respuestas a lo planteado al inicio a través de las interrogantes, los objetivos y presupuestos. 
La dimensión ética de la investigación se desarrolló a partir de cinco principios: confidencialidad de las respuestas, libertad de participar o no en la investigación, respeto por la forma de pensar de cada participante, sinceridad al contestar y el consentimiento.

\section{Resultados CuANTITATIVOS}

Es el universalismo-preocupación el tipo motivacional de valor que encabeza la jerarquía en esta investigación y se refiere al compromiso por la igualdad, justicia y protección de las demás personas. En segundo lugar, la benevolencia-fiabilidad, la cual se refiere a ser confiable y responsable con las demás personas de un grupo. "Colocamos el universalismo-preocupación social junto a la benevolencia-fiabilidad, porque ambos enfatizan el compromiso con el bienestar de otros" (Schwartz, et al., 2012, p. 670). A partir de lo propuesto por la teoría para los resultados generales, el alumnado está más interesado en las demás personas que en sí mismo; el compromiso con la sociedad en general, en primer lugar, y sus relaciones al interno de los grupos, en segundo lugar.

En último lugar de la jerarquía, se ubica recursos de poder y en penúltimo lugar poder-dominación. Lo que comprueba este hecho es que, en la propuesta, ambos se ubican adyacentemente y puede notarse la polaridad respecto a los más puntuados según el círculo motivacional. El poder ha sido encontrado con mayores puntuaciones en otros contextos, por ejemplo, Japón.

Los dominios, cuya diferencia se inclina a mayor presencia en las mujeres, son: autodirección-pensamiento, seguridad social, humildad, universalismo-preocupación, universalismo-tolerancia. Esto perfila el análisis hasta los valores de orden superior; se puede afirmar que las mujeres presentan mayores diferencias en los dominios orientados al focus social, al colectivismo, la preocupación por la otredad, por la sociedad en general.

Las diferencias que manifiestan mayor presencia en los varones son los dominios de: recursos de poder y poder dominación. Estos dominios se ubican en la autopromoción, que, a su vez, se inclina hacia el individualismo. Esta bipolaridad de preferencias en la variable sexo manifiesta la distinción de la propuesta de Schwartz. De hecho, esta propuesta es heredera de toda una tradición de estudios que buscan las dos facetas polarizadas en diferentes sociedades.

Las mujeres demuestran una tendencia más arraigada al colectivismo, dato bastante parecido al encontrado por Castiglione et al. (2014). Las mujeres manifiestan esta tendencia por ser más prosociales y abiertas al cambio. Estas personas autoras descubren que los varones adolescentes dan mayor prioridad al poder. Con esto parece volverse a estereotipos de género, al encontrarse tendencias en las cuales las mujeres son las encargadas del hogar, por ejemplo, amas de casa, cuidadoras de los hijos e hijas, mientras el hombre es quien manda en el hogar.

Con la aplicación de un análisis de varianza (ANOVA), los resultados muestran que en la variable edad, los dominios que presentan diferencias intergrupales son: libertad de acción $(\mathrm{F}=0.05 \mathrm{p}<0.05)$, hedonismo $(\mathrm{F}=0.000 \mathrm{p}<0.05), \operatorname{logro}(0.021 \mathrm{p}<0.05)$ y benevolencia-cuido $(0.038 \mathrm{p}<0.05)$. Sin embargo, en el desarrollo de un análisis intergrupal de comparación múltiple por rangos de edad y con la aplicación de la prueba de Tukey, existen diferencias significativas únicamente en el dominio de hedonismo. Con esta prueba se ha encontrado la correlación que a mayor edad, menor presencia de hedonismo.

Es en la seguridad social donde existe diferencia significativa en la variable naturaleza (confesionalesaconfesionales), que proyecta una mayor presencia de este dominio en los centros aconfesionales $(t=.024$ $\mathrm{p}<0.05)$, al igual que el universalismo-naturaleza $(\mathrm{t}=.016 \mathrm{p}<0.05)$. Aunque en los demás dominios, al observar la jerarquía por grupos individuales, puede notarse la preferencia, cuando se comparan las medias con $t$ de student no existen diferencias significativas que lleven a afirmar la presencia mayor de esos dominios entre grupos.

Los resultados generales casi se replican en su totalidad para ambos tipos de centros, públicos y privados. El hedonismo y logro se ubican en el cuarto lugar de ambos. Ningún tipo motivacional de valor presenta 
diferencia significativa en esta variable. Aunque se puede observar un poco de variación entre la posición de los dominios de los tres primeros lugares de ambos grupos, por ejemplo, si el análisis se hace teniendo en cuenta los valores de orden superior que representan los dominios, puede observarse oposición igual que en el resultado general; la autotrascendencia y la autopromoción se manifiestan como polos contrarios, con la salvedad de la conformidad con las reglas, ya que es común su posición para ambos grupos.

\section{Resultados cualitativos}

Al buscar significado de los valores para la población participante, se encuentra que lo más importante para ellos es Dios. Consideran que el creer en Dios es el inicio de muchos valores, aunque no necesariamente, pertenecer a una denominación religiosa sea sinónimo de no creer en Él o de ser buenas personas. Creer en Dios va relacionado con la búsqueda de ser buenas personas que a su vez se relaciona con los valores. Los valores hacen buenas a las personas.

Las experiencias con los valores están marcadas a partir del compañerismo, pues la convivencia cercana les posibilita establecer relaciones de amistad. Esta concatenación de experiencias estudiantiles hace que su vida esté cargada de vivencias en las que se resaltan personas, hechos, modelos de vida a partir de los cuales atribuir significados y una orientación de sus vidas desde lo percibido.

Reconocen que han vivido situaciones específicas en las que los valores se ponen en práctica: "A mí me tocó vivir esto y me ha enseñado el valor de la honestidad, responsabilidad, compromiso y transparencia porque una sola papeleta que faltara iba en contra nuestra y manchar todo el trabajo" (E.3 comunicación personal, 15 de agosto de 2019). También han admirado el buen ejemplo de algunas maestras y maestros, cuyos aportes no necesariamente coinciden con lo académico, sino con el buen trato y los consejos que les han dado.

Una de las personas entrevistadas (E.5) se ha convertido en un modelo adolescente a seguir en términos de práctica de valores. Los valores se evidencian en las conductas de personas concretas, en sus acciones; los valores los entienden como si estuvieran fundidos a las personas, hablan de ella. "Pues algo que me ha dejado marcado siempre es nuestro compañero E.5, que él ha marcado de siempre la humildad, respeto hacia los demás. Nunca nos ha fallado, siempre nos ha apoyado y nos ha dejado marcados" (GF. 2 comunicación personal, 16 de agosto de 2019).

\section{Valores y bondad}

Los valores que más reflejan la bondad de una persona son: tolerancia, respeto, humildad, perseverancia y paciencia. Resaltan que el valor que ven como un productor de buenas personas es la humildad. Estos valores darían el calificativo de buena a la persona a quien los practique con un ingrediente más: la fe. "Si yo amo a Dios voy a tener buenos valores entonces podríamos decir que la fe es el complemento" (E.4, comunicación personal, 14 de agosto de 2019). El principio de la bondad es Dios. Las personas humildes, tolerantes y que no crean prejuicios, son buenas personas.

\section{Antivalores y maldad}

Una persona mala es alguien que humilla a las demás, es prepotente, soberbia e irrespetuosa. El estudiantado participante hace la identificación de una persona mala a partir de antivalores, falta de valores y todo se origina por la falta de compasión.

\section{Clarificación de valores}

La humildad es el indiscutible valor elegido. Humildad como sinónimo de bondad. Ante esta propuesta metodológica de la clarificación de valores, los estudiantes no han vacilado en dar su jerarquía. En la esta misa, de forma unánime, ha aparecido la humildad, que es anhelada tanto para la vida profesional como para la formación humana. El conflicto generado sobre este valor con resultados diferentes en cada acercamiento, queda explicitado en la discusión.

Valores y vida profesional 
La interpretación se hace a partir de los verbos resaltados en su discurso. Personas humildes, profesionales humildes, buenas personas. Desde un acercamiento hermenéutico a los verbos resaltados, el estudiantado participante manifiesta una unión indudable entre el sí y la otredad.

Según Escalante (2010), los verbos que expresan "estados, acción y juicio están asociados a los valores de los estudiantes" (p. 32). Estos verbos, asociados a los relatos desde las entrevistas, presentan una estructura cultural interpretativa. A partir de esta técnica, la búsqueda de verbos significativos ayuda a encontrar hacia dónde los valores que perfilan a las personas. Entre esos verbos están: vivir, enseñar, aprender, valorar, conseguir, diligenciar, marcar, apoyar, tener, creer, ayudar, entender, alcanzar, ser, acercar y conectar. Se interpreta que los valores significan a partir del anhelo de servir; su vida profesional al servicio de las demás personas como un anhelo futuro.

\section{Discusión}

\section{Lo prosocial}

En los resultados globales, los primeros lugares en la jerarquía han sido dominios prosociales. Estos resultados se parecen a lo encontrado por Redondo y Oliva (2017) en la posición de valores de orden superior. Pero estas personas autoras trabajaron con docentes, población adulta en el contexto español. En la presente investigación, el hallazgo desde ambos acercamientos coincide, al menos, en lo que respecta a la benevolenciacuido, cuya jerarquía ha sido ubicada en la tercera posición en el acercamiento cuantitativo, y se ha mencionado una relación estrecha intrafamiliar desde lo cualitativo. Esto apunta a considerar, en esta población, una orientación hacia lo social más como significado que como estadística.

Por otro lado, conforme la edad aumenta, también se incrementa la preferencia por la benevolencia-cuido, los lazos emocionales y el amor por la familia, pero disminuye la benevolencia-fiabilidad. La necesidad de ser aquellas personas en quien otras pueden confiar disminuye (Schwartz et al., 2012). Este resultado también refleja una especialización femenina más fuerte en la benevolencia-cuido que en la fiabilidad. Aun así, las mujeres tienen una inclinación más fuerte al colectivismo que los varones, quienes están más arraigados al individualismo.

\section{Sobre la edad y el hedonismo}

El Hedonismo presenta diferencia significativa en la variable de edad. En contextos como el español, se ha encontrado correlación entre este dominio y el uso del internet. En lo que respecta al contexto hondureño, Redacción WEB (2017), a partir de una entrevista realizada a una persona experta en redes sociales, ha advertido la adicción a estas. Se afirma que el 27 por ciento de quienes usan redes sociales está entre los 12 y los 20 años de edad. En un estudio confirmatorio sobre esta relación se puede comprobar si este dominio, que ha mostrado diferencia significativa en la variable edad, en realidad correlacione con la frecuencia de uso de las redes sociales. Lo interesante del hallazgo en el contexto hondureño es que no se encuentra en el primer lugar de la jerarquía, en consideración de la etapa en la que se encuentra la población objeto de estudio, para quienes como se ha manifestado son otras las motivaciones.

\section{El asunto del poder}

Medina (2015) interpreta el dominio de poder como una motivación caracterizada por el apego al estatus social, el prestigio y control o el dominio de las demás personas y recursos. Junto a logro, ambos se enfocan 
en la autoestima. El estudiantado, al ser heredero de una tradición que ha considerado el dinero, la riqueza, la autoridad y el dominio como algo no tan deseado, mantiene esta visión y se aferra a lo que han recibido de sus mayores. Sobre este dominio, como se ha mencionado, se han encontrado mayores puntuaciones en países orientales.

\section{La adyacencia y las posibles correlaciones}

De acuerdo a los resultados, las variables externas con las que correlacionarían los dominios más puntuados según Schwartz, en esta investigación, son: la familia como prioridad, las personas inmigrantes deberían tener los mismos derechos y la propuesta de que "el gobierno debería reducir las diferencias de ingresos", que ha encontrado significancia predicha con universalismo-preocupación (Schwartz et al., 2012, p. 682).

Un hallazgo, tal vez extrañado para los centros confesionales, ha sido la ausencia de la benevolencia en el primer lugar de su jerarquía. Personas autoras como García et al. (2016) han buscado adyacencia entre el dominio benevolencia y la confesionalidad, al argumentar que este dominio debería ser un calificativo para los centros confesionales. En el contexto del presente estudio, es en la seguridad social que existe diferencia significativa en esta variable, pues se proyecta una mayor presencia de este dominio en los centros aconfesionales $(t=.024 \mathrm{p}<0.05)$ al igual que el universalismo-naturaleza $(t=.016 \mathrm{p}<0.05)$. El sentido de buscar resaltar esta relación radica en toda la enseñanza de amor al prójimo, con la cual se presenta la confesionalidad. Es posible que el impacto de esta enseñanza, en este contexto, genere otro tipo de convicciones y poco interés en el servicio al prójimo.

\section{Dios, los valores y la formación humana}

El significado de los valores en esta área está supeditado a Dios, pero no un Dios de la convicción, es el Dios enseñado, heredado. La deseabilidad social sobresale en esta semántica valórica de origen divino. La virtud teologal de la fe vendría a ser la fuente de los valores morales. Se llega a ser buena persona por fe. El proceso catequético de inculcación de valores entra al juego. Hay una cultura bastante arraigada en la fe. La formación humana no es concebida sin la fe en Dios. Los valores significan en la medida que tengan a Dios como fuente.

Pero esta visión religiosa de los valores también tiene otra faceta: La satanización del dinero y del poder; de hecho, las aspiraciones en la vida son vistas como ambiciones que se deben rechazar. Una persona que tiene a Dios en su vida no debe aspirar a los bienes, al dinero o al disfrute, pues quien nace pobre, vive pobre, los ricos están contados y el dinero y el placer son prohibidos. Además, el pobre gozará en la otra vida. Al contrario, las personas de bien deben practicar virtudes como la humildad. ¿Cuál es el peso de la tradición en esta realidad? Como dominio valórico no parece ser tan importante, considerando la posición en la jerarquía, pues es de los menos puntuados; posición 16 en la tabla.

En la teoría de Schwartz, la bondad se concreta a partir de la benevolencia-cuido. Aunque en ningún momento esta propuesta hace alusión a Dios como origen del bien, los resultados generales establecen una tendencia hacia este dominio, que, junto al universalismo-preocupación, ha sido de los más puntuados. La particularidad del dominio, inclinado hacia la otredad, es coincidencia entre lo estadísticamente confirmado y lo expresado en las entrevistas. El estudiantado participante afirma que en el servicio a otros y otras se expresa la bondad que viene de Dios.

\section{La humildad}

Junto a la visión de Dios como fuente de valores, amor al prójimo y paz, la humildad está incrustada en la mentalidad del estudiantado, incluso desde la interpretación de la importancia de los valores para su vida 
profesional. En primer lugar, no se debe olvidar el origen religioso del término humildad. El significado atribuido, para quienes participaron en el estudio, se encamina por el lado de la modestia, asociado con un tipo de servicio, caridad y servicio desinteresado. Sin embargo, ser un término valorado por el grupo le otorga un valor agregado, pues ser una persona profesional humilde es ya poseer un perfil ante las demás. Este valor se convierte en algo deseable. Además, la creencia arraigada acerca de que quien es humilde, es bueno, le agrega importancia y deseabilidad a este valor.

Por otro lado, la humildad crea modelos de personas virtuosas. Como se ha mencionado en los resultados, E. 5 ha sido un estudiante muy bien considerado por sus pares, un modelo de buena persona. Pero, lejos de su éxito académico, es más considerado como un modelo de vida porque es humilde. Valores como este se encarnan en docentes memorables que han proyectado un testimonio digno de imitar y pueden convertirse en modelos de buenas personas, tema que aparece en la interpretación de los valores: los modelos de vida para la población adolescentes.

Personas autoras como Korres (2015) reconocen que "los adolescentes, en pleno proceso de búsqueda personal, encuentran en las figuras mediáticas modelos de identificación y comparación que participan en la definición de su propia identidad y la construcción de sus valores" (p. 1). Estas personas autoras, al consultar a la población adolescente sobre por qué elige personajes que admiran y son modelos a seguir, descubrieron que el grupo de jóvenes tiene en cuenta: el buen humor del personaje, la personalidad (jamás se rinde), el amor por la familia, la inteligencia, el atractivo físico, la riqueza y éxito, el trabajo y la originalidad. Medrano et al. (2009) también analizaron los valores que los medios de comunicación le presentan al público joven y encontraron que estos valores se inclinan hacia la diversión, la amistad, la exaltación del grupo, búsqueda de sensaciones, libertad, competitividad, la transgresión o la naturalidad. Lo interesante es que en ninguno de los dos estudios citados aparece la humildad, ni encarnada en un personaje, ni como oferta propagandística.

Desde la visión cuantitativa, la humildad no parece resaltar estadísticamente. Los ítems que miden la humildad como un dominio motivacional son tres. En una escala de 1 a 6, en el cuestionario de valores retratados, se consulta cuánto se parece la persona de la que se habla en el ítem a la persona encuestada, en consideración de que, para esa persona descrita en los ítems, es importante ser humilde, estar satisfecha con lo que tiene, no pedir más y no pensar que merece más que las demás personas. En la puntuación general, este dominio se ubicó en la posición número once, adyacente con el dominio de conformidad, sin mostrar diferencias significativas en cuanto sexo o edad. Lo único que resalta es la adyacencia de la propuesta. Aparte de eso, lo relevante aparece en el acercamiento cualitativo.

Con esta ambivalencia se descubre que los ítems no necesariamente reflejan lo que para el estudiantado significa ser humilde. No es que los ítems planteados sean insuficientes, es que este valor se expresa, significa y tiene peso cuando alguien lo practica. Por ello, los valores, en general, adquieren sentido en la conversación. La humildad cualifica (Frondizi, 1972) y la cualificación se expresa (Medina, 2015).

\section{El interés por los valores}

Se ha pensado la profesión como un servicio. Su jerarquía como profesionales está constituida por valores como la humildad, el respeto, la amabilidad, la compasión, la sencillez, la tolerancia, el servicio, la responsabilidad, la honestidad y la solidaridad. Estos valores son sinónimo de añoranzas. No es que el estudiantado se sienta humilde, sencillo o servicial, sino es que lo piensa como futuras personas profesionales. El tiempo está en la base, el éskaton hebreo se hace presente. 


\section{La dimensión prosocial como ideación vocacional a partir de los valores}

Muchas veces decimos necesitamos esto, nos podemos dar un lujo y es cierto, podemos trabajar para darnos ciertos lujos, pero debemos de tomar en cuenta que en este mundo hay personas que nos necesitan y que no deberíamos quedarnos en nuestra zona de confort y no solo hacer para mí, para mi familia, si no que de vez en cuando no está demás poder ayudar a los demás, poder prestar un poco de lo que nosotros tenemos. Entonces opino que sí, que todos tenemos que tomar un tiempo en nuestra vida y decir: puedo ayudar, entonces debo hacerlo (E. 3, comunicación personal, 15 de agosto de 2019).

La población estudiantil entrevistada interpreta que sin valores no hay amor al prójimo. Este tema tan abordado en nuestros días busca entender la conducta prosocial de la población adolescente. Desde la teoría de Schwartz, esta dimensión característica del grupo adolescente se aborda con el colectivismo, dominios como la benevolencia y el universalismo también han sido resaltados.

Es un cúmulo de emociones que caracteriza a la población estudiada. Las experiencias narradas desde el amor por su familia, pasando por los modelos de vida, la admiración de personas que dan un buen ejemplo como los maestros que logran trazar un perfil profesional, el amor demostrado por sus padres y madres, y hasta las modelos que quisieran imitar, la mención de valores es abundante. El interés mostrado por ellos se interpreta a partir de su significado, crea una esperanza fijada en un estereotipo de persona inspirada en valores.

\section{Conclusiones}

\section{Los tipos motivacionales de valor: una mirada desde Schwartz}

De acuerdo a esta investigación, las preferencias de valor en el estudiantado se dirigen hacia la búsqueda de igualdad, justicia y protección de las demás personas. Sin embargo, por la naturaleza de la teoría utilizada desde el diseño cuantitativo, no puede afirmarse que estos o aquellos son los valores presentes, de modo fijo e inalterable en la población estudiantil del último año en Ciencias y Humanidades del área urbana de Choluteca, en consideración de que se han buscado preferencias de valor, no valores específicos con el acercamiento cuantitativo.

En segundo lugar, la población estudiantil encuestada prefiere ser confiable, responsable y buscar el bienestar de las personas del grupo al que pertenecen. Por otro lado, lo menos importante para ellas y ellos es el ejercicio del poder sobre otras personas y el control de recursos materiales y sociales para generar poder. En la variable edad, se encuentra una correlación negativa: a mayor edad, menor presencia de hedonismo y, entre más jóvenes, mayor presencia. Según los resultados, las mujeres están orientadas al focus social, al colectivismo, la preocupación por la otredad y los varones se inclinan más al individualismo.

El estudiantado de la modalidad a distancia prefiere el logro a diferencia de la modalidad presencial. Pero, en una comparación con el hedonismo, ambos tienen un comportamiento parecido en la variable edad: aumenta a los 21 años y decae a los 23 .

Los resultados para la variable del tipo de centro (públicos-privados) no fueron tan diferentes con los resultados generales. No se observa una diferencia marcada en las preferencias. El universalismopreocupación y los dos tipos de benevolencia se ubican en los primeros lugares de las preferencias.

El hedonismo está más marcado en los centros bilingües que en los no bilingües, pues este último lo ubican en el quinto lugar y el primero, en segundo. Las posibilidades de correlaciones de este tipo motivacional de valor con otras variables podrían ser significativas.

Contrario a lo encontrado en otros contextos, cuyos resultados están alineados a la inclinación hacia las demás personas (benevolencia) que deberían tener los centros educativos confesionales, en la presente 
investigación es en la seguridad social y el universalismo-naturaleza donde hay diferencia significativa a favor de los centros aconfesionales. Es posible, entonces, que este dominio en el contexto hondureño no necesariamente este asociado a la confesionalidad y todo lo que ello implica en cuanto a la enseñanza litúrgica, bíblica, pastoral, etc.

\section{Significado e interpretación de los valores: formación humana y vida profesional}

El significado que el estudiantado entrevistado otorga a los valores se forja a partir de las vivencias y experiencias que de ellos ha tenido en diferentes espacios. Habla desde su formación humana, es decir, desde la experiencia que tiene a partir de su sociedad estudiantil, la convivencia, la importancia de los valores en su jerarquía de prioridades y su capacidad para diferenciar a una persona buena de una mala, desde los valores o antivalores que ven reflejados en ella. Desde estas subcategorías, los valores significan bondad y construcción de personas virtuosas y modelos de vida, cuyo valor resaltado ha sido la humildad.

Por otro lado, su formación humana se posiciona a partir de su fe en Dios y solamente de Él puede brotar la bondad, pues, para el grupo, el principio de la bondad es Dios, el Dios heredado y es a partir de este ser supremo que los valores adquieren sentido, Dios como fuente de todo valor. Pero no es el Dios de las iglesias, es más su creencia en Dios. Sugiere una religiosidad no practicante, una especie de convencionalismo.

Por otro lado, anhelan ser humildes y sobre este valor se debe cimentar cualquier proyecto humano. La humildad en este sentido se convierte en la marca del modelo de vida, admiran a personas no tanto por su nivel académico, sino por la humildad que reflejan y por eso quieren ser como ellas.

La concepción humana es, por tanto, la base para la vida profesional. El estudiantado no se ve desligado de esta unión entre ambos estados: formación humana y vida profesional; traducido a una frase: solo una persona humilde puede ser una persona profesional humilde, por tanto, un ejemplo digno de imitar, pues humildad es igual a bondad. Mencionan sus valores antes que otro tipo de cualidad. Se convierten en modelos exigibles al igual que la justicia. Los valores significan en la medida que alguien los practique. El interés en este campo se manifiesta en el reflejo al admirar lo deseado; si ven que una persona es humilde manifiestan su anhelo de cercanía con ella a partir de su admiración y el deseo de su amistad.

Por otro lado, la interpretación del interés que tiene el estudiantado por los valores para su vida profesional, se circunscribe a la formación humana. Esto es, no la considera la vida profesional desligada de la formación humana, sino que la práctica de valores establece un perfil profesional inspirado en una formación humana que tenga como su centro la vivencia de valores.

En este sentido, el interés mostrado por los valores se interpreta a partir de la deseabilidad por ser profesionales humildes que, como se ha dicho, equivale a decir que serán profesionales humildes como una visión futura. Una persona profesional humilde es una persona profesional buena; una persona humilde puede llegar a ser una persona profesional humilde y, por tanto, una persona profesional buena. Pero es un interés por el futuro, es decir, consideran que los valores son principios sumamente necesarios para el ejercicio de su profesión en un futuro. El presente es únicamente de preparación.

Por tanto, el perfil axiológico del estudiantado lo constituye la preocupación por la otredad y el anhelo de justicia; lo que no integra ese perfil es la búsqueda de poder. Igual que en otros contextos, la búsqueda de placer también se manifiesta según la edad. Los centros confesionales y su inclinación por la preocupación por el otro no es algo marcado en este contexto. La creencia en Dios es también característico del perfil axiológico del estudiantado; creencia muy arraigada en dicha población y marca la concepción de esta. Finalmente el valor de la humildad significa bondad y el anhelo de lograrlo se proyecta en una perspectiva profesional futura. 


\section{RECOMENDACIONES}

Con la presente investigación han surgido nuevos horizontes para estudios posteriores a partir de la teoría de Schwartz en el contexto hondureño, los cuales podrían estar orientados a buscar correlación con algún comportamiento específico a ejemplo de otros contextos, cuyos planteamientos actuales se conducen por esa dirección o con otras variables, como las que se han propuesto de acuerdo a los resultados generados. Los dominios de valor y la lectura, la violencia, la migración, la deshonestidad académica en los centros escolares, el bullying y un sinnúmero de problemáticas que cada región conoce, podrían ser posibilidades para investigaciones.

Se recomienda un seguimiento enfocado en esta propuesta teórica, pues daría un mayor sustento en el carácter predictor de esta variable, porque ha demostrado la presencia de los dominios valóricos transculturales en el contexto hondureño.

El planteamiento cualitativo también ha sido una exploración desde el diseño fenomenológico con matices interpretativos. Varios proyectos investigativos podrían gestarse a partir de este tipo de acercamientos. Podrían establecerse planteamientos con poblaciones mucho más grandes, sea en la educación media, en los diferentes ciclos de primaria y hasta en la universidad. Esto también establece una idea de las posibles rutas a seguir en estudios posteriores.

\section{El interés por la otredad.}

Es posible que los impulsos orientados al servicio de las demás personas no estén del todo explotados en estudiantes de estas edades, al menos como un hecho real. Por tanto, se recomienda fortalecer esta dimensión. No restringir la enseñanza a un aula de clases, es necesario encarar al estudiantado con su realidad, con las poblaciones más necesitadas y desde allí fomentar el compromiso por la transformación social.

\section{El valor de la humildad}

Se recomienda centrar una investigación en este tema. La humildad es un valor anhelado por el estudiantado, aunque los ítems que lo miden en la teoría de Shwartz no han sido la preferencia más puntuada. Cuando se habla de apreciación, definición y la subjetividad expresada al este, la situación cambia y aparece la ambivalencia axiológica. De todas formas, no se debe desconocer el valor agregado que tiene una persona humilde; la identificación de una persona humilde con una persona buena y todo el perfil deseado que otorga este valor a quien se califique como humilde. Futuras investigaciones al respecto podrían adquirir diferentes matices.

\section{Los valores y la familia}

¿Cómo conjugar una propuesta de formación en valores teniendo como centro a la familia? La reflexión en torno a este aspecto podría producir elementos importantes. Dada la situación desfigurada de las familias hondureñas, este desfase no es impedimento para que la población estudiantil encuestada mencione a su familia como lo más preciado. Los lazos familiares son muy fuertes. Esta concepción familiar no se restringe a sus padres, madres, hermanas o hermanos, sino que es una extensión que incluye amigos cercanos como familia. Se recomienda una pedagogía moral que no desconozca este elemento. 


\section{El fomento del amor hacia el prójimo en colegios confesionales}

En cuanto a tendencia no se encuentra relación entre el dominio de benevolencia y la confesionalidad. Como un anhelo de ayudar, el significado dado a partir de la compasión y la solidaridad expresada, puede afirmarse que existe en un nivel no diferenciado respecto de colegios aconfesionales. En el fondo, el reclamo a la enseñanza de las diferentes denominaciones hace posible que esta misma impacte en otras dimensiones, menos el amor al prójimo y prójima, el fomento de la bondad, la benevolencia y la empatía con el sufrimiento de la otredad. Esta dimensión es débil. Es recomendable enderezar los programas hacia la promoción o desarrollo de la sensibilidad y el ejercicio de la caridad. Si se retoma esta recomendación y se encuentra eco en el estudiantado (con base en la etapa de vida inclinada hacia aspectos relacionados con esta propuesta), se estaría estableciendo un elemento característico para centros educativos de esta naturaleza.

¿Cómo formar personas profesionales comprometidas con las necesidades de las demás personas, orientadas a la coincidencia entre vocación y profesión, entre trabajo y servicio?

Con el desarrollo de una investigación sobre personas (que incluya docentes) memorables. Quienes participaron en el estudio han mencionado algo de las personas que admiran. Sin embargo, sería interesante saber qué valores tienen en realidad esas personas, aparte de humildad. Desde allí se podría construir un perfil axiológico desde la vida profesional o desde la formación humana y la construcción de la humanidad.

\section{Referencias bibliográficas}

Allport, G., Vernon, P. y Lindzey, G. (1931). Test de valores. https://bit.ly/3aTjeSk

Alto Comisionado de las Naciones Unidas para los Refugiado [ACNUR]. (2018). Impacto de la violencia en 220 centros educativos de Tegucigalpa. https://www.acnur.org/5bf4a1c14.pdf

Benítez, G. (2015). Ensayo del libro: ¿Qué son los valores? De Risieri Frondizi. Fondo de cultura económica.

Camps, V. (2010) El declive de la ciudadanía. La construcción de una ética pública. Editorial y Distribuidora, S.A.g

Castiglione, C., Rampullo, A. y Licciardello, O. (2014). High School Students Value System. Social and Behavioral Sciences, 141, 1330-1334.https://doi.org/10.1016/j.sbspro.2014.05.229

Cedeño, N. (2012). La investigación mixta, estrategia andragógica fundamental para fortalecer las capacidades intelectuales superiores. Res non verba, 17-36. https://bit.ly/3acXZe8

Comisión Económica para América Latina [CEPAL]. (2019). Panorama Social de América Latina. CEPAL. https:/ /tinyurl.com/y2tzwgbo

Cortina, A. (2009). La educación y los valores. Biblioteca Nueva.

Cortina, A. (2013). Ética discursiva y Educación en valores. Universidad de Valencia. https://caredu.files.wordpress.c om/2010/04/adela-cortina-etica-discursiva-y-educacion-en-valores.pdf

De Waal, F. (2014). El bonobo y los diez mandamientos, en busca de la ética de entre los primates. (A. García, Trad.). Tusquets editores.

Escalante, E. (2010). Valores en estudiantes universitarios, una investigación hermenéutica. Liber [online], 16(1), 27-38. https://n9.cl/q8sj5

Frondizi, R. (1972). ¿Qué son los valores? (3ra ed.). Fondo de Cultura Económica.

Fullat, O. (2005). Valores y Narrativa, Axiología educativa de Occidente. Universitat de Barcelona.

García, R., Muchotrigo, M. G. y Manzanares, E. L. (2016). Jerarquía de valores entre estudiantes de secundaria de colegio religioso y colegio laico en Lima. LIBERAVIT, 22(2), 229-238. http://www.scielo.org.pe/pdf/liber/v2 2n2/a10v22n2.pdf

Goodwin, R., Realo, A., Kwiatkowska, A., Luu, L. y Nizharadze. (2002). Values and Sexual Behavior in Central and Eastern Europe. Journal of Health Psycology, 7(1), 45-56. https://doi.org/10.1177/1359105302007001651 
Gutierrez, K. (2016). Abordaje fenomenológico de la praxis investigativa y cosmovisiones de los docentes de un sistema de educación a distancia. Investigación y postgrado, 31(1), 101-130. http://revistas.upel.edu.ve/index.php/revin post/article/view/5091

Hofstede, G. (2011). Dimensionalizing Cultures: The Hofstede Model in Context. Online Readings in Psychology and Culture, 2(1), 1-26. https://doi.org/10.9707/2307-0919.1014

Imhoff, D. y Brussino, S. (2013). Estudio Exploratorio de las Características Psicométricas del Portrait Values Questionnaire en el Contexto de Córdova. Argentina. Universidad Nacional de Córdova-Argentina.Revista Colombiana de Psicología, 22(1), 135-149. https://revistas.unal.edu.co/index.php/psicologia/article/view/205 $\underline{94}$

Inglehart, R. (1994). Modernización y post-modernización: la cambiante relación entre el desarrollo económico, cambio cultural y político. En J. Díez y R. Inglehart (Eds.). Tendencias mundiales de cambio en los valores sociales ypoliticos. (pp. 63-107). FUNDESCO.

Iocoboni, M. (2009). Las neuronas espejo. Katz editores.

Jonas, H. (1995) El principio de responsabilidad: ensayo de una ética para la civilización tecnológica. Herder.

Kasof, J., Chen, C., Himsel, A. y Greenberger, E. (2007). Values and Creativity. Creativity Research Journal, 19, 105-122. https://www.researchgate.net/publication/247807756_Values_as_Creativity

Kohlberg, L. (1992). Psicología del desarrollo moral. Desclée De Brouver.

Korres, O. (2015). Los valores percibidos por los adolescentes de Bizkaia en su personaje televisivo de ficción seriada. Una aproximación desde los modelos de Schwartz y Hall-Tonna [Tesis de doctorado inédita]. Universidad de Deusto. https://doi.org/10.13140/RG.2.2.28942.18246

Medina, D. (2007). Estudio de la conceptualización de valor y las estrategias de transmisión y/o construcción de valores utilizadas por los maestros en centros públicos y privados del primer ciclo del nivel Básico. Santo Domingo. Ciencia y Sociedad, 32(3), 364-420. https://www.redalyc.org/pdf/870/87032301.pdf

Medina, E. (2015). Diseño de una escala multifactorial basada en la teoría de Schwartz para medir los valores personales en las organizaciones peruanas [Tesis de doctorado inédita]. Universidad de Sevilla. https://idus.us.es/bitstream /handle/11441/39446/version\%20final.pdf?sequence =\&isAllowed =y

Medrano, C., Cortés, A. y Palacios, S. (2009). Los valores personales y los valores percibidos en la televisión: un estudio con adolescentes. Revista Electrónica Interuniversitaria de Formación del Profesorado, 12(4), 55-66. https://ww w.redalyc.org/pdf/2170/217014941005.pdf

Moreno, J. (2006). Valores, actitudes hacia el alcohol y consumo en adolescentes varones. Limite, I(13), 195-211. $\underline{\mathrm{h}}$ ttps://www.researchgate.net/publication/26506171

Naval, C. (2001) Los nuevos paisajes éticos: el debate en la educación en valores en la actualidad. En C. Vilanou y E. Collelldemont (Eds.). Historia de la educación en valores. (pp. 261-276). Desclée de Brouwer.

Ortega y Gasset, J. (1961). Introducción a una estimativa ¿qué son los valores? Alianza Editorial, (versión digital) htt ps://bit.ly/3aPgV2L

Piaget, J. (1984). El criterio moral en el niño. (N. Vidal, Trad.) Martínez Roca. https://www.nodo50.org/filosofem/I MG/pdf/piage_el_criterio_moral_en_el_nino.pdf

Puig J. (2003). La construcción de la personalidad moral. Ediciones Paidós Ibérica.

Redacción WEB (2017, 28 de octubre). Experto afirma que los hondureños son adictos a las redes sociales. El tiempo. https://tiempo.hn/hondurenos-adictos-redes-sociales/2/

Redondo, V. y Oliva, Á. J. (2017, agosto). Valores sociales del profesorado de infantil, primaria y secundaria: influencia de la edad y el sexo. [Sesión de congreso]. XXX Congreso Internacional de Pedagagia Social. Universidad Nacional de Educación a distancia, Sevilla, España, 110-123. https://idus.us.es/handle/11441/74296

Rodríguez, G., Flores, G. y García, E. (1999). Metodología de la investigación cualitativa. ALJIBE.

Rokeach, M. (1973). The Nature of human values. Social Forces, 53(4), 650-660. https://doi.org/10.1093/sf/53.4.659

Rorty, R. (2004) Derechos humanos, racionalidad y sentimentalismo. En Th. Abraham, A. Badiou, R. Rorty (Eds.). Batallas éticas. (pp. 59-80). Nueva Visión. 
Sánchez, P. (2016). Adaptación del Cuestionario de Valores Retratados (PVQ-RR) con estudiantes de primer ingreso de la sede Rodrigo Facio de la Universidad de Costa Rica [Tesis de Licenciatura], Universidad de Costa Rica, sede de Occidente. https://portal.so.ucr.ac.cr/sites/default/files/documents/Tesis\%20PVQ\%20Final.pdf

Sandoval, M. (2007). Sociología de los valores y juventud. Última década, 17, 95-118. https://scielo.conicyt.cl/pdf/ udecada/v15n27/art06.pdf

Scheller, M. (2001). ÉTICA, Nuevo ensayo de fundamentación de un personalismo ético. Caparrós Editores. https://w ww.salud.gob.ec/wp-content/uploads/2016/12/5.1-Scheler-Etica-71-78.pdf

Schwartz, S. (1992). Universals in the content and structure of values: theoretical advances and empirical tests in 20 countries. Advances in Experimental Social Psychology, 25, 1-65, https://bit.ly/3pwcfFf

Schwartz, S. (2012). An Overview of the Schwartz Theory of Basic Values. Online Readings in Psychology and Culture, 2(1), 1-20. https://doi.org/10.9707/2307-0919.1116

Schwartz, S. y Bilsky, W. (1987). Toward al psychological structure of human values. Journal of Personality and Social Psychology, 53(3), 550-562. https://doi.org/10.1037/0022-3514.53.3.550

Schwartz, S., Cieciuch, J., Vecchione, M. y Davidov, E. (2012). Refining the Theory of Basic Individual Values. Journal of Personality and Social Psycology, 53(3) 663-688-. https://bit.ly/2MjmUEP

Spranger, E. (1928). Types of men. Target Training International.

Turró, G. (2001) La ética de la responsabilidad: Hans Jonas. En C. Vilanou y E. Collelldemont (Eds.) Historia de la educación en valores, (pp. 239-246). Desclée de Brouwer.

Thiebaut, C. (2009) La revolución del pensamiento político y moral. En M. Garrido, L. Valdéz y L. Arenas (Eds.) El legado filosófico y científico del siglo XX. (pp. 605-616). Ediciones Cátedra.

Triandis, H. (2001). Individualism-Collectivism and Personality. Journal of Personality, 69(6), 907-924.https://doi. org/10.1111/1467-6494.696169

\section{INFORMACIÓN ADICIONAL}

Cómo citar: Osorto, S. F, y Soriano, O. A. (2022). Preferencias y significado de valores en estudiantes del bachillerato. Revista Educación, 46(1). http://doi.org/10.15517/revedu.v46i1.45402 